\title{
Direct admission to the coronary care unit by the ambulance service for patients with suspected myocardial infarction
}

\author{
N Prasad, A Wright, K J Hogg, F G Dunn
}

\begin{abstract}
Background-Direct access to the coronary care unit (CCU) for general practitioner (GP) referred cases of suspected acute myocardial infarction (AMI) (fast track admission) substantially reduces the time to thrombolysis. Until now, this policy has been confined to GP referrals.

Objectives-To determine the time taken to admission to CCU under the fast track policy (ambulance referrals and GP referrals) and the time taken to start administration of thrombolytics (ambulance referrals, GP referrals, and accident and emergency referrals).

Methods-Fast track admission policy was extended to include referrals from ambulance personnel who respond to emergency service calls. Ambulance personnel referred cases were also examined to see if they were referred appropriately to the CCU.

Results-100 ambulance personnel referrals and 260 GP referrals to CCU with chest pain were studied. Forty accident and emergency referrals who had AMI requiring thrombolysis were also studied. In the ambulance referred group the time to admission from phone call was a median of 10 minutes (range 2 to 45), a saving of 30 minutes compared with GP referrals (median 40 minutes, range 2 to 217). The median diagnostic electrocardiogram (ECG) to thrombolysis time was longer in the accident and emergency referrals with AMI than either ambulance referrals or GP referrals admitted under the fast track policy. Diagnostic ECG to thrombolysis time: accident and emergency 50 minutes (range 15 to 385); ambulance referrals median 33 minutes (range 6 to 69); GP referrals median 29.5 minutes (range 5 to 110 minutes); ( $p=0.056$ accident and emergency compared with ambulance referrals, $p<0.002$ accident and emergency compared with GP referrals). Of 100 ambulance referrals 52 patients exhibited symptoms suggestive of ischaemic heart disease (confirmed AMI, unstable angina, and angina) and a further 18 patients were required to stay in CCU for other cardiac problems. Thus a total of 70 (70\%) were considered appropriate compared with 155 of 260 (55.8\%) GP referred cases.
\end{abstract}

Department of Cardiology, Stobhill Hospital, Glasgow G21 3UW, UK

Correspondence to: Dr Dunn.

Accepted for publication 4 July 1997
Conclusions-Extending the fast track admission policy to ambulance personnel reduces delay to admission for patients with suspected MI without adversely affecting the appropriateness of admissions. (Heart 1997;78:462-464)

Keywords: direct admission to coronary care; time to thrombolysis; ambulance personnel

The benefits of thrombolytic therapy for acute myocardial infarction (AMI) are maximised by the earliest possible administration of these agents. ${ }^{1}$ In our hospital we have provided direct access to the coronary care unit (CCU) for general practitioner (GP) referred cases of suspected AMI (fast track admission) for a number of years. We have shown that this policy substantially reduces the time to thrombolysis (by 44 minutes) in those patients with an AMI because the delays caused by assessment in the casualty department are avoided. ${ }^{2}$ Until now, this policy has been confined to GP referrals. However as a considerable number of patients are admitted directly to hospital via the ambulance service it is also important that delay is minimised in these patients. The fast track admission policy was therefore extended to include referrals from ambulance personnel who respond to emergency service calls. The purpose of this study was to evaluate this extension to our policy by examining the time taken to admission and the time taken to start administration of thrombolysis therapy in those with AMI referred by ambulance personnel. We also examined ambulance personnel referred cases to see if they were referred appropriately to the CCU.

\section{Methods}

Our hospital has access to coronary care via a backdoor from which ambulances can transfer patients directly into coronary care. Initial assessment of patients referred directly to CCU is made in a CCU bed by the admitting medical registrar or senior house officer (SHO). The registrar/SHO assesses the needs of the admission and decides whether coronary care, acute medical ward care or discharge home is appropriate. Standard criteria are applied for thrombolysis including: a history consistent with AMI, with characteristic electrocardiographic (ECG) abnormalities including: $1 \mathrm{~mm}$ ST elevation in two standard ECG leads or $2 \mathrm{~mm}$ of ST elevation in two 


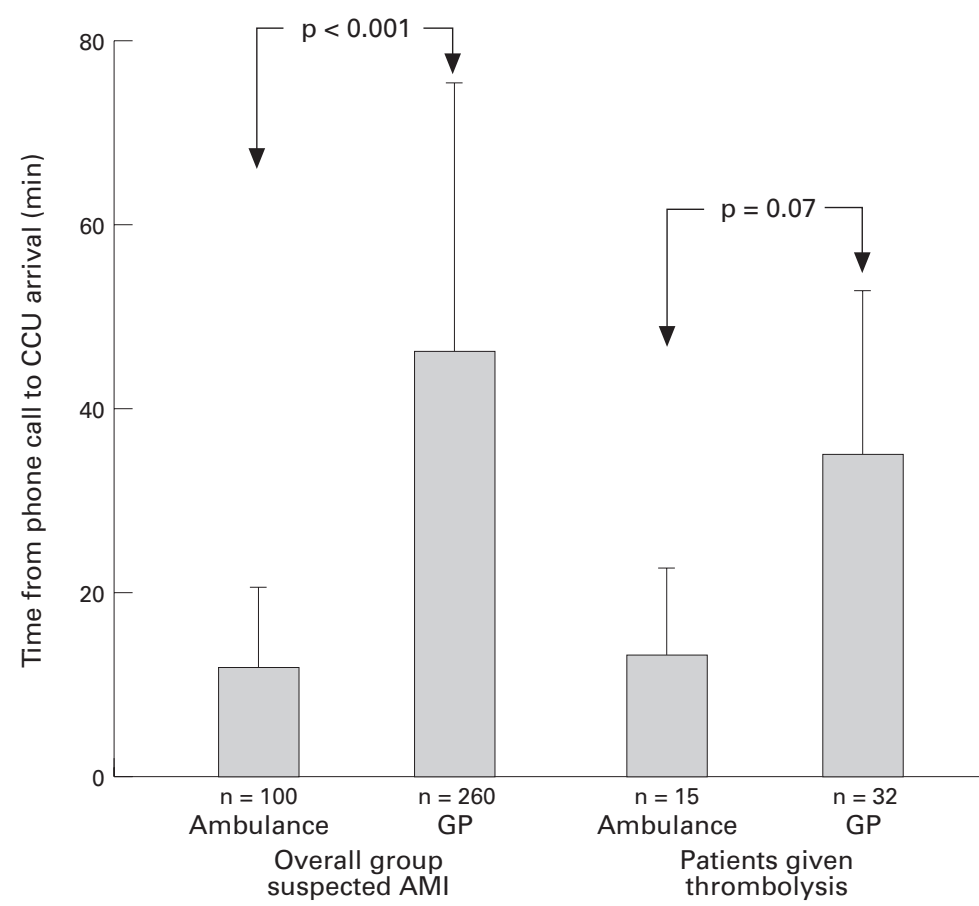

Figure 1 Time taken from phone call to admission to coronary care (CCU) in suspected acute myocardial infarction ( $A M I)$ and those patients requiring thrombolytic therapy once admitted. Ambulance referrals arrive earlier to CCU than GP referrals (median values; error bars, standard deviations).

precordial chest leads or the presence of left bundle branch block.

All ambulance personnel serving our catchment zone were informed of the system for fast track admission. If the ambulance staff were summoned by an emergency service call to a patient and they suspected an AMI they would call CCU requesting direct admission. No other additional training was given to the ambulance personnel. The delay to admission and final diagnosis were analysed in 100 consecutive admissions during 1995 referred via the ambulance service and were compared with GP admissions to CCU over a six month period in 1995. The time from phone call to admission, the diagnostic ECG to thrombolysis time, and the final diagnosis were compared between the two groups. We also continued to monitor the diagnostic ECG to thrombolysis time in those with an AMI who presented to accident and emergency during the same six months in 1995. Statistical analysis by unpaired Student's $t$ test was used to examine differences between groups.

Table 1 Confirmed diagnosis in the patients referred to coronary care by ambulance personnel and general practitioners

\begin{tabular}{lcccc}
\hline & Confirmed $M I$ & $\begin{array}{l}\text { Unstable } \\
\text { angina }\end{array}$ & Angina & Other \\
\hline Ambulance service referrals & 21 & 22 & 9 & 48 \\
Total admissions ( $=100)$ & 20 & 15 & 1 & 18 \\
Admissions to CCU $(\mathrm{n}=54)$ & 1 & 7 & 8 & 30 \\
Transfer to ward ( $=46)$ & & & & \\
General practitioner referrals & 60 & 46 & 43 & 111 \\
Total admissions $(\mathrm{n}=260)$ & 54 & 30 & 10 & 22 \\
Admissions to CCU $(\mathrm{n}=116)$ & 6 & 16 & 33 & 89 \\
Transfer to ward $(\mathrm{n}=144)$ & & & & \\
\hline
\end{tabular}

\section{Results}

A total of 100 ambulance personnel referrals (median age 62 years (range 26 to 86 ), 69 men) and $260 \mathrm{GP}$ referrals (median age 64 years (range 28 to 94 ), 150 men) were studied. Figure 1 shows the time from phone call to admission to CCU. In the ambulance referred group this was a median of 10 minutes (range 2 to 45), a saving of 30 minutes compared with GP (median 40 minutes, range 2 to 217) referrals in patients suspected to have had an AMI. Those subjects subsequently given thrombolysis in the ambulance group also arrived to hospital 25 minutes earlier than the patients given thrombolysis in the GP group (fig 1). Once admitted to CCU the time to the initiation of thrombolysis from diagnostic ECG in those with an AMI was similar in both groups (ambulance referrals $(\mathrm{n}=15)$ median $33 \mathrm{~min}$ utes (range 6 to 69$)$, GP referrals $(n=32)$ median 29.5 minutes (range 5 to 110 ); NS). In subjects who presented to accident and emergency having sustained an AMI during the study ( $\mathrm{n}=40,30$ men, median age 55 years, range 42 to 88 ) the median diagnostic ECG to thrombolysis time was longer at 50 minutes (range 15 to 385 ) ( $\mathrm{p}=0.056$ accident and emergency compared with ambulance referrals, $\mathrm{p}<0.002$ accident and emergency compared with GP referrals).

Table 1 shows that the final diagnoses of these admissions are broadly similar between the groups. We had concerns that a higher number of inappropriate admissions may be directed to the CCU becaause of the policy change, however this was not the case. Of the 100 ambulance referrals 52 patients exhibited symptoms suggestive of ischaemic heart disease (table 1), (confirmed AMI, unstable angina, and angina) and a further 18 patients were required to stay in CCU for other reasons. Thus a total of $70(70 \%)$ were considered appropriate compared with 155 of 260 $(55.8 \%)$ GP referred cases (table 1 ).

The uptake of the service was initially slow as it was when the service was first introduced for GP referrals. This has now stabilised and we now expect around 10 admissions per month from the ambulance service.

\section{Discussion}

Extending the fast track admission policy to include referrals by the ambulance service has a substantial benefit to patients with suspected AMI. Avoiding delays in casualty departments gains these critically ill cardiac patients the same advantage of earlier specialist attention as those directed to CCU by GPs. The ambulance group have an additional advantage in that they receive hospital care faster than if a GP is called first, there being a reduction in delay of 30 minutes compared with GP referrals. Patients with AMI admitted by the fast track policy whether ambulance or GP referred continue to receive thrombolysis earlier than patients presenting to accident and emergency with a shorter diagnostic ECG to thrombolysis time by around 20 minutes. 
Our fast track admission policy to CCU of patients not only improves care for patients but reduces the workload for the casualty department. From this study we would recommend that those patients who suspect that they are having an AMI should call an ambulance after discussing the symptoms with their GP on the telephone. The ambulance personnel could then decide if CCU care or review in casualty is required. This would reduce transfer time to CCU and possibly the number of house calls for GPs. However the increased number of patients passing through CCU will increase workload in this department.
It is clear from this study that extending the fast track admission policy to ambulance personnel saves on the time to admission for AMI patients and that ambulance personnel can be relied on to decide about the appropriateness of the admission to CCU.

The authors express their gratitude to all ambulance personnel who participated in the conduct of this study.

1 Fibrinolytic Therapy Trialists (FTT) Collaboration Group. Lancet 1994;343:311-22.

2 Burns JMA, Hogg KJ, Rae AP, Hillis WS, Dunn FG. Impact of policy of direct admission to a coronary care unit on the use of thrombolytic treatment. Br Heart $\mathcal{F} 1989 ; 61: 322-5$. 